\title{
Uniformly exponential growth and mapping class groups of surfaces
}

\author{
J. W. Anderson, J. Aramayona, and K. J. Shackleton
}

\begin{abstract}
We show that the mapping class group (as well as closely related groups) of an orientable surface with finitely generated fundamental group has uniformly exponential growth. We further demonstrate the uniformly nonamenability of many of these groups.
\end{abstract}

\section{Uniformly exponential growth}

The main purpose of this note is to demonstrate that the mapping class group of an orientable surface with finitely generated fundamental group has uniformly exponential growth. This result is new for surfaces of genus at least one, with the exception of the closed surface of genus two, and can be viewed as removing a possible avenue for showing that such mapping class groups are not linear. In this sense, our work is similar in spirit to the recent work of Brendle and Hamidi-Tehrani $[\mathrm{BH}]$.

We go on to show that closely related groups of homotopy classes of homeomorphisms of surfaces, as well as analogous groups of automorphisms of free groups, also have uniformly exponential growth. We remark that, while the linearity of most surface mapping class groups is an open question, most automorphism groups of free groups are known not to be linear. Specifically, as noted in Brendle and HamidiTehrani $[\mathbf{B H}]$, it is known that $\operatorname{Aut}\left(F_{n}\right)$ is not linear for $n \geq 3$ and $\operatorname{Out}\left(F_{n}\right)$ is not linear for $n \geq 4$, whereas both $\operatorname{Aut}\left(F_{2}\right)$ and $\operatorname{Out}\left(F_{2}\right)$ are linear. We also demonstrate the uniform non-amenability of many of the groups considered in this note; see Section 2

We begin by reviewing some basic definitions. For a survey of exponential growth and uniformly exponential growth, we suggest the article of de la Harpe $[\mathbf{H}]$ and the references contained therein.

Let $G$ be a finitely generated group, and let $S$ be a finite generating set for $G$. The length $\ell_{S}(g)$ of an element $g \in G$ is the least integer $k$ so that $g$ can be expressed as $g=s_{i_{1}} \cdots s_{i_{k}}$, where each $s_{i_{j}} \in S \cup S^{-1}$. We define the length of the identity element of $G$ to be 0 . Let

$$
B_{S}(n)=\left\{g \in G \mid \ell_{S}(g) \leq n\right\}
$$

2000 Mathematics Subject Classification. MSC 20F65 (primary), 20F38 (secondary). 
be the ball of radius $n$ about the identity element in $G$, and let $\left|B_{S}(n)\right|$ be the cardinality of $B_{S}(n)$.

The exponential growth rate $\omega(G, S)$ of $G$ with respect to $S$ is defined to be

$$
\omega(G, S)=\lim _{n \rightarrow \infty} \sqrt[n]{\left|B_{S}(n)\right|}
$$

(This limit exists, due to the submultiplicativity $\ell_{S}(g h) \leq \ell_{S}(g) \ell_{S}(h)$ of the length function on $G$.) The group $G$ has exponential growth if $\omega(G, S)>1$ for some (and hence for every) finite generating set $S$. Note that if $G$ has a free subgroup of rank 2 , then $G$ has exponential growth, though not conversely.

We can also remove the dependence on particular generating sets by considering

$$
\omega(G)=\inf _{S} \omega(G, S),
$$

where the infimum is taken over all finite generating sets $S$ of $G$. The group $G$ is said to have uniformly exponential growth if $\omega(G)>1$. For examples of groups of exponential growth that do not have uniformly exponential growth, see Wilson $[\mathbf{W}]$.

We make use of the following proposition from de la Harpe $[\mathbf{H}]$.

Proposition 1 (from Proposition 2.3 of de la Harpe $[\mathbf{H}]$ ). If $G$ is a finitely generated group and if $G^{\prime}$ is a quotient of $G$, then $\omega(G) \geq \omega\left(G^{\prime}\right)$.

We use the following result of Shalen and Wagreich $[\mathbf{S W}]$ to show the uniformly exponential growth for certain groups related to the mapping class group (see Corollary 7 and Corollary 8).

Lemma 2 (from Corollary 3.6 of Shalen and Wagreich $[\mathbf{S W}]$ ). Let $G$ be a finitely generated group and let $H$ be a finite index subgroup of $G$. If $H$ has uniformly exponential growth, then $G$ has uniformly exponential growth.

REMARK 3. To the best of our knowledge, it is not yet known whether the converse of Lemma 2 holds. It seems the main difficulty lies in singling out an extended generating set for $G$ from one for $H$ to give uniform embeddings on Cayley graphs. In this note, such issues represent only a minor inconvenience. A positive answer would prove uniformly exponential growth to be a commensurability invariant; we conjecture that this should be true.

Eskin, Mozes, and Oh [EMO] consider the question of uniformly exponential growth for finitely generated subgroups of $\operatorname{GL}(n, \mathbf{C})$. In particular, they prove the following.

Theorem 4 (Corollary 1.4 of Eskin, Mozes, and Oh [EMO]). Let $\Gamma$ be a finitely generated subgroup of $\mathrm{GL}(n, \mathbf{C})$. The following are equivalent:

- $\Gamma$ is not virtually nilpotent;

- $\Gamma$ is of uniformly exponential growth;

- $\Gamma$ is of exponential growth.

The main tool we use is the following result, which should be viewed as an immediate corollary of Theorem 4.

TheOREM 5. Let $G$ be a finitely generated group, and suppose that for some $n \geq 2$ there exists a homomorphism $\rho: G \rightarrow \mathrm{GL}(n, \mathbf{C})$ whose image $\rho(G)$ is not virtually nilpotent. Then, $G$ has uniformly exponential growth. 
Proof. Since $\rho(G)$ is finitely generated and not virtually nilpotent, Theorem 4 yields $\omega(\rho(G))>1$ (that is, $\rho(G)$ has uniformly exponential growth). Since $\rho(G)$ is the homomorphic image of $G$, it is a quotient of $G$. Proposition 1 yields that $\omega(G) \geq \omega(\rho(G))>1$, and so $G$ has uniformly exponential growth.

It is known that virtually nilpotent groups have polynomial growth (and conversely, by work of Gromov $[\mathbf{G}]$ ), whereas groups containing a free group of rank at least two have exponential growth.

We are now ready to define the mapping class group of an orientable surface with finitely generated fundamental group. We cite the survey article of Ivanov $[\mathbf{I}]$ as our main reference for the mapping class group and its properties.

Let $\Sigma$ be a closed orientable surface of genus $g \geq 1$, and let $P$ be a finite set of marked points on $\Sigma$, where $n=|P| \geq 0$. The mapping class group $\mathcal{M}(\Sigma, P)=\mathcal{M}_{g, n}$ is the set of homotopy classes of orientation preserving homeomorphisms $f: \Sigma \rightarrow \Sigma$ for which $f(P)=P$, where the homotopies are required to keep each point of $P$ fixed. Note that the elements of $\mathcal{M}_{g, n}$ can permute the points of $P$. (We can also view $\mathcal{M}_{g, n}$ as the mapping class group of a surface of genus $g$ with $n$ punctures.)

There is a natural surjective homomorphism from $\mathcal{M}_{g, n}$ to $\operatorname{Symm}(n)$, the symmetric group on $n$ letters, given by restricting the action of $\mathcal{M}_{g, n}$ to $P$. The kernel $\mathcal{P} M_{g, n}$ of this homomorphism is the pure mapping class group, which is the subgroup of $\mathcal{M}_{g, n}$ fixing every element of $P$. Note that $\mathcal{P} M_{g, n}$ is a subgroup of finite index in $\mathcal{M}_{g, n}$.

Now suppose $n=|P|>0$ and let $p \in P$. By forgetting the marked point $p$ we find that every homeomorphism $f: \Sigma \rightarrow \Sigma$ fixing $P$ pointwise induces a homeomorphism $f^{\prime}: \Sigma \rightarrow \Sigma$ fixing $P \backslash\{p\}$ pointwise. In this way we get a surjective homomorphism $\mathcal{P} M_{g, n} \rightarrow \mathcal{P} M_{g, n-1}$; in particular, we see that $\mathcal{P} M_{g, n}$ homomorphically surjects onto the mapping class group $\mathcal{P} \mathcal{M}_{g, 0}=\mathcal{M}_{g, 0}=\mathcal{M}_{g}$ of the surface $\Sigma$ with no marked points.

The extended mapping class group $\mathcal{M}_{g, n}^{ \pm}$is the group of homotopy classes of all homeomorphisms of $\Sigma$ fixing $P$ setwise, and is a degree 2 extension of $\mathcal{M}_{g, n}$.

We can make similar definitions in the case that $\Sigma$ is allowed to have nonempty boundary. Let $\Sigma$ be a compact orientable surface of genus $g \geq 1$ and let $P$ be a finite set of $n=|P| \geq 0$ marked points in the interior of $\Sigma$. Let $m$ denote the number of components of $\partial \Sigma$. The mapping class group $\mathcal{M}_{g, n, m}$ is the group of homotopy classes of orientation preserving homeomorphisms of $\Sigma$. The pure mapping class group $\mathcal{P} M_{g, n, m}$ is the subgroup of $\mathcal{M}_{g, n, m}$ consisting of those elements that permute neither the components of $\partial \Sigma$ nor the elements of $P$. As before, $\mathcal{P} M_{g, n, m}$ is a subgroup of finite index in $\mathcal{M}_{g, n, m}$. The extended mapping class group $\mathcal{M}_{g, n, m}^{ \pm}$is the group of homotopy classes of all homeomorphisms of $\Sigma$, and is a degree 2 extension of $\mathcal{M}_{g, n, m}$.

We note that for a compact orientable surface $\Sigma$ with non-empty boundary, there is a natural surjective homomorphism from $\mathcal{P} \mathcal{M}_{g, n, m}$ to $\mathcal{P} \mathcal{M}_{g, n, 0}=\mathcal{P} \mathcal{M}_{g, n}$, obtained by gluing discs to all the boundary components of $\Sigma$ and extending the homeomorphisms of $\Sigma$ across these discs; this is discussed in detail in Theorem 2.8.C of Ivanov $[\mathbf{I}]$.

We are now ready for the main result of this note.

THEOREM 6. For $g \geq 1$, the groups $\mathcal{M}_{g}$ and $\mathcal{M}_{g}^{ \pm}$have uniformly exponential growth. 
ProOF. Let $\Sigma$ be a closed surface of genus $g \geq 1$ and consider the mapping class group $\mathcal{M}_{g}$ of $\Sigma$. Recall that $\mathcal{M}_{g}$ is finitely generated. We show the result for $\mathcal{M}_{g}$; Lemma 2 then yields the result for $\mathcal{M}_{g}^{ \pm}$, which is a degree 2 extension of $\mathcal{M}_{g}$ as we noted above. The natural action of $\mathcal{M}_{g}$ on $H_{1}(\Sigma, \mathbf{Z})=\pi_{1}(\Sigma) /\left[\pi_{1}(\Sigma), \pi_{1}(\Sigma)\right]$ yields a surjective homomorphism $\rho: \mathcal{M}_{g} \rightarrow \operatorname{Sp}(2 g, \mathbf{Z})$. Since $\operatorname{Sp}(2 g, \mathbf{Z})$ contains $\mathrm{Sp}(2, \mathbf{Z}) \cong \mathrm{SL}(2, \mathbf{Z})$ as a subgroup and since $\mathrm{SL}(2, \mathbf{Z})$ contains a $\mathbf{Z} * \mathbf{Z}$ subgroup, we see that $\rho\left(\mathcal{M}_{g}\right)=\operatorname{Sp}(2 g, \mathbf{Z})$ is not virtually nilpotent. Hence, by Theorem 5 , we see that $\mathcal{M}_{g}$ has uniformly exponential growth; by Lemma 2 , we see that $\mathcal{M}_{g}^{ \pm}$ has uniformly exponential growth.

[To see that $\operatorname{Sp}(2 g, \mathbf{Z})$ contains $\operatorname{Sp}(2, \mathbf{Z}) \cong \operatorname{SL}(2, \mathbf{Z})$, recall that $\operatorname{Sp}(2 g, \mathbf{Z})$ is defined to be the group of $2 g \times 2 g$-matrices preserving a non-degenerate, skewsymmetric bilinear form. If we take the form $\mathbf{q}(\mathbf{x}, \mathbf{y})=x_{1} y_{2}-x_{2} y_{1}+\cdots+x_{2 g-1} y_{2 g}-$ $x_{2 g} y_{2 g-1}$, then any block diagonal matrix of the form

$$
\left(\begin{array}{cc}
A & \mathbf{0}_{2,2 g-2} \\
\mathbf{0}_{2 g-2,2} & I_{2 g-2}
\end{array}\right)
$$

preserves $\mathbf{q}$ and hence lies in $\operatorname{Sp}(2 g, \mathbf{Z})$, where $A$ lies in the group $\operatorname{Sp}(2, \mathbf{Z})$ preserving the quadratic form $\mathbf{q}_{0}(\mathbf{x}, \mathbf{y})=x_{1} y_{2}-x_{2} y_{1}, \mathbf{0}_{s, t}$ is the $s \times t$ matrix of zeroes, and $I_{k}$ is the $k \times k$ identity matrix.]

We saw before that there is a surjective homomorphism from $\mathcal{P} M_{g, n}$ to $\mathcal{P} M_{g}=$ $\mathcal{M}_{g}$, and that $\mathcal{P} M_{g, n}$ has finite index in $\mathcal{M}_{g, n}$. Further, $\mathcal{M}_{g, n}$ is finitely generated. Proposition 1 and Lemma 2 then yield the following corollary.

Corollary 7 . For $g \geq 1$ and $n>0$, the groups $\mathcal{P} M_{g, n}, \mathcal{M}_{g, n}$ and $\mathcal{M}_{g, n}^{ \pm}$have uniformly exponential growth.

We also saw that, in the case of surfaces with boundary, there is a homomorphism from $\mathcal{P} M_{g, n, m}$ to $\mathcal{P} M_{g, n}$, and that $\mathcal{P} M_{g, n, m}$ has finite index in $\mathcal{M}_{g, n, m}$. Further, $\mathcal{M}_{g, n, m}$ is finitely generated. We thus get the following result, again using Proposition 1 and Lemma 2 .

Corollary 8. For $g \geq 1, m>0$, and $n \geq 0$, the groups $\mathcal{P} M_{g, n, m}, \mathcal{M}_{g, n, m}$ and $\mathcal{M}_{g, n, m}^{ \pm}$have uniformly exponential growth.

For $g=0$, the methods we use here do not apply. We note that when $n \geq 4$, it is a remarkable result of Bigelow $[\mathbf{B i}]$ and Krammer $[\mathbf{K r}]$ that each $\mathcal{M}_{0, n}$ is linear (and not virtually nilpotent) and hence has uniformly exponential growth by Theorem 4. For $g=2$ and $n=0$, it is a result of Bigelow and Budney [BB] and of Korkmaz $[\mathbf{K o}]$ that $\mathcal{M}_{2,0}$ is linear and hence has uniformly exponential growth, again by Theorem 4 .

\section{Uniform non-amenability}

We note that the same argument we have given for the uniformly exponential growth of the mapping class groups $\mathcal{M}_{g}$ also yields their uniform non-amenability. Following Arzhantseva et al. [ABLRSV], given a finitely generated group $G$ with finite generating set $X$, define the inner boundary of a finite subset $A$ of $G$ to be

$$
\partial_{X}(A)=\left\{a \in A \mid a x \notin A \text { for some } x \in X \cup X^{-1}\right\} \text {. }
$$


The uniform Følner constant of $G$ is then

$$
\operatorname{Fol}(G)=\inf _{X} \inf _{A} \frac{\left|\partial_{X}(A)\right|}{|A|}
$$

where the outer infimum is taken over all (finite) generating sets $X$ of $G$ and the inner infimum is taken over all finite subsets $A$ of $G$. We say that $G$ is uniformly non-amenable if $\operatorname{Fol}(G)>0$.

We make use of the following results about uniform non-amenability, which are the analogues of the similar results for uniformly exponential growth. The first notes how uniform non-amenability behaves under quotients.

Proposition 9 (from Theorem 4.1 of Arzhantseva et al. [ABLRSV]). Let $G$ be a finitely generated group, and let $N$ be a normal subgroup of $G$. Then, $\operatorname{Fol}(G) \geq \operatorname{Fol}(G / N)$.

The second describes the uniform non-amenability of linear groups.

TheOREM 10 (from Corollary 1.2 of Breuillard and Gelander $[\mathbf{B r G}]$ ). Let $\Gamma$ be a finitely generated subgroup of $\mathrm{GL}(n, \mathbf{C})$. Assume that $\Gamma$ is not amenable. Then, $\Gamma$ is uniformly non-amenable.

We now consider the uniform non-amenability of mapping class groups.

THEOREM 11. The groups $\mathcal{M}_{g}$ are uniformly non-amenable for all $g \geq 1$.

Proof. Proposition 9 states that if $G^{\prime}$ is a quotient of the finitely generated group $G$, then $\operatorname{Fol}(G) \geq \operatorname{Fol}\left(G^{\prime}\right)$; this takes the place of Proposition 1. Theorem 10 states that finitely generated non-virtually nilpotent linear groups over any field are uniformly non-amenable; this takes the place of Theorem 4, and the analogue of Theorem 5 for uniform non-amenability then follows immediately. The proof of Theorem 6 then proceeds to show that the groups $\mathcal{M}_{g}$ are uniformly non-amenable for all $g \geq 1$.

Applying Proposition 9 then shows that any groups that surject onto one of the $\mathcal{M}_{g}$ for $g \geq 1$ are also uniformly non-amenable; see Corollary 7 and Corollary 8 . This leaves out $\mathcal{M}_{g}^{ \pm}$, as the analogue of Lemma 2 is not known to hold for uniform non-amenability.

\section{Automorphisms of free groups}

Analogous results hold for the automorphism group $\operatorname{Aut}\left(F_{n}\right)$ of the free group $F_{n}$ of rank $n$ and for the outer automorphism group $\operatorname{Out}\left(F_{n}\right)$, the quotient of $\operatorname{Aut}\left(F_{n}\right)$ by the group of inner automorphisms.

THEOREM 12. For $n \geq 2$, the groups $\operatorname{Aut}\left(F_{n}\right)$ and $\operatorname{Out}\left(F_{n}\right)$ have uniformly exponential growth.

Proof. Begin with $\operatorname{Out}\left(F_{n}\right)$. The natural action of $\operatorname{Out}\left(F_{n}\right)$ on $F_{n} /\left[F_{n}, F_{n}\right]=$ $\mathbf{Z}^{n}$ yields a surjective homomorphism $\rho: \operatorname{Out}\left(F_{n}\right) \rightarrow \operatorname{GL}(n, \mathbf{Z})$. Since $\operatorname{GL}(n, \mathbf{Z})$ is finitely generated and is not virtually nilpotent (as $\operatorname{SL}(2, \mathbf{Z}) \subset \operatorname{SL}(n, \mathbf{Z}) \subset$ $\operatorname{GL}(n, \mathbf{Z}))$, Theorem 5 yields that $\operatorname{Out}\left(F_{n}\right)$ has uniformly exponential growth.

That $\operatorname{Aut}\left(F_{n}\right)$ has uniformly exponential growth follows from Proposition 1, since $\operatorname{Out}\left(F_{n}\right)$ is a quotient of $\operatorname{Aut}\left(F_{n}\right)$. 
As above, the same proof (with appropriate substitutions, as in the proof of Theorem 11) shows that for $n \geq 2$, the groups $\operatorname{Aut}\left(F_{n}\right)$ and $\operatorname{Out}\left(F_{n}\right)$ are uniformly non-amenable.

Acknowledgements. Parts of this paper were written during visits of the second author to the Bernoulli Center, Lausanne, and the third author to the Tokyo Institute of Technology. Both authors wish to express their gratitude to these institutions. The third author was supported by an EPSRC studentship. The first author would also like to thank Gareth Jones for helpful conversations.

\section{References}

[ABLRSV] G. N. Arzhantseva, J. Burillo, M. Lustig, L. Reeves, H. Short, E. Ventura, Uniform non-amenability, Adv. Math. 197 (2005), 499-522.

[Bi] S. J. Bigelow, Braid groups are linear, J. Amer. Math. Soc. 14 (2001), 471-486.

[BB] S. J. Bigelow, R. D. Budney, The mapping class group of a genus two surface is linear, Algebr. Geom. Topol. 1 (2001), 699-708.

$[\mathrm{BH}]$ T. E. Brendle, H. Hamidi-Tehrani, On the linearity problem for mapping class groups, Algebr. Geom. Topol. 1 (2001), 445-468.

[BrG] E. Breuillard, T. Gelander, Cheeger constant and algebraic entropy of linear groups, Int. Math. Res. Not. 56 (2005), 3511-3523.

[EMO] A. Eskin, S. Mozes, H. Oh, On uniform exponential growth for linear groups, Invent. Math. 160 (2005), 1-30.

[H] P. de la Harpe, Uniform Growth in Groups of Exponential Growth, Geom. Dedicata 9 (2000), $1-17$.

[G] M. Gromov, Groups of polynomial growth and expanding maps, Inst. Hautes Etudes Sci. Publ. Math. 53 (1981), 53-73.

[I] N. V. Ivanov, Mapping class groups, in Handbook of Geometric Topology, edited by R. J. Daverman and R. B. Sher, Elsevier Science B. V., London, 2002, 523-633.

[Ko] M. Korkmaz, On the linearity of certain mapping class groups, Turkish J. Math. 24 (2000), $367-371$.

[Kr] D. Krammer, Braid groups are linear, Ann. of Math. 155 (2002), 131-156.

[SW] P. B. Shalen and P. Wagreich, Growth Rates, $\mathbf{Z}_{p}$-Homology, and Volumes of Hyperbolic 3-Manifolds, Trans. Amer. Math. Soc. 331 (1992), 895-917.

[W] J. S. Wilson, On exponential growth and uniformly exponential growth for groups, Invent. Math. 155 (2004), 287-303.

School of Mathematics, University of Southampton, Southampton SO17 1BJ, EngLAND

E-mail address: j.w.anderson@soton.ac.uk

Mathematics Institute, University of Warwick, Coventry CV4 7AL, England

E-mail address: jaram@maths.warwick.ac.uk

Department of Mathematical and Computing Sciences, Tokyo Institute of TechNOlOGy, 2-12-1 O-OKayama, Meguro-Ku, TOKYo, 152-8552 Japan

E-mail address: kjs2006@alumni.soton.ac.uk 